\title{
Parallel Processing and Scheduling Techniques Applied to the Quality Control of Bill Sheets
}

\author{
J. $\mathbf{M}^{\mathrm{a}}$. Sebastián ${ }^{\dagger}$, F. Torres ${ }^{\dagger}$, O. Reinoso ${ }^{\dagger}$, J. L. Bello ${ }^{\ddagger}$, E. Barroso ${ }^{\ddagger}$ \\ (†) Dep. Automática, Electrónica e Informática Industrial. (U.P.M.) \\ (†) Fábrica Nacional de Moneda y Timbre
}

\begin{abstract}
Automatic quality control examination of banknotes constitutes an important research challenge for the main printing organizations in the world. It is worth to note that presently there is no commercial product with an acceptable performance, in spite of the efforts spent during last ten years. The product complexity lies in the combination of several printings with the purpose of achieving hard to counterfeit banknotes. The printing system that is necessary for banknotes manufacturing, causes significant differences between several printed sheets in similar conditions. However, in spite of these differences, while respecting certain tolerances, the banknotes are accepted. The required quality of the product demands an exhaustive inspection of the bill sheets. Nowadays the quality control is carried out by manual turn over of the bill sheet, reaching a speed of 400 sheets/hour. It has been developed a system that works out an automatic inspection at a rate of 3.600 sheets/hour.
\end{abstract}

\section{1: Introduction}

On one hand, the system must be capable of inspecting at high rate according to the production speed, and on the other hand the quality control of bill sheets must be consistent and always with the same inspections conditions, eliminating the subjective judgment and variability of manual inspection. These two reasons introduce the development of the system for an automatic quality examination. This system is composed by:

- A mechanical system handling sheets. The purpose of this system lies in placing sheets precisely on the acquisition image zones to take the front side images.

- An inspection system putting together several computer vision techniques and parallel processing, in order to get the issues described in section 2 .

\section{1: Inspection system hardware.}

Inside the computer system the following physical components can be distinguished: illumination system, camera system, control module and the modules of image processing.

The acquisition table, placed in a mechanical base, has its own illumination system and an appropriate module for reading images. This module is made by twelve cameras that allow to obtain a resolution of $0.3 \mathrm{~mm}$. per pixel.

The illumination system and the cameras of each one of the acquisition tables are confined in a watertight compartment that prevents undesirable influences in the uniform illumination. The camera distribution in the acquisition table allows, through a telescopic structure, to fit the different existing denominations( differents sheets size). The cameras are based in CCD technology with interline transfer of half resolution (756 x 581 pixels). The video signal is CCIR standard and allows to get 25 frames per second. The cameras contain two synchronism types, external and internal. All of them are equipped with a $28 \mathrm{~mm}$. lens that allows to get the images without distortion.

The image processing module is made up of four acquisition and processing image boards Image Series of Matrox. Each board manages three cameras. Both modules are managed by an additional control module.

\section{2: Inspection system software.}

Each hardware module is connected to a specific software module. In this way it can be distinguished between: (1) the software created for the control module that manages not only the control of all the tasks that the computer system must realize in every moment but also the study of the inspection results (Figure 1); and (2) the software created for the acquisition and processing image modules that allows, in a lower level, to manage the 
specific tasks for the parallel execution of the inspection algorithm.

The control module constitutes the interface between the global system and the user. This module, according to the parameters decided by the user, depending of the features of the denomination, generates the inspection lines. Besides it manages the information of the

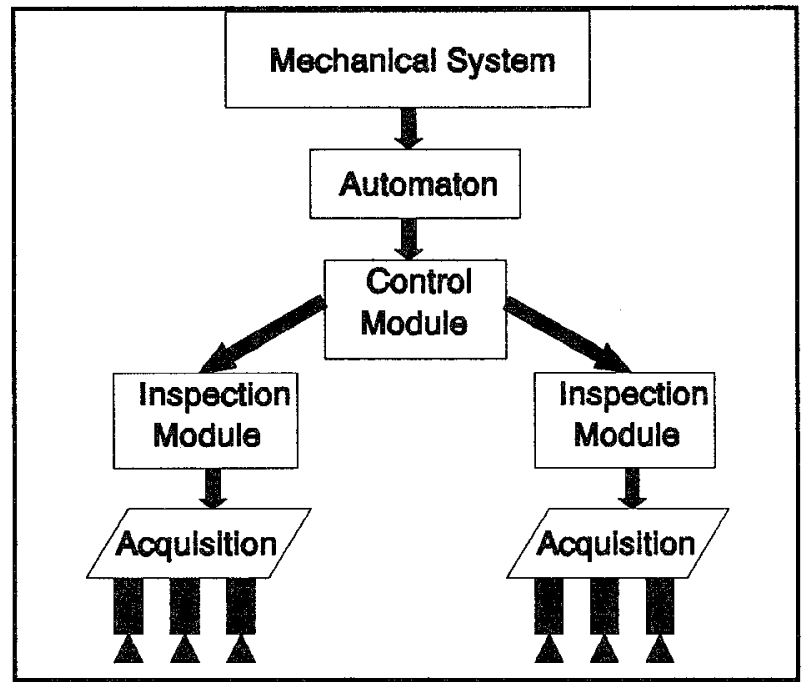

Figure 1

inspection results. In this way it generates statistics about the defective sheets, production control, ..., preserving the results of the last evaluated set.

In the following sections the significative aspects of the software created for the inspection modules will be detailed.

\section{2: Inspection Algorithm.}

The inspection and the quality control of the sheets is made over each banknote in the sheet. In this way, the specific parameters associated with the inspection algorithm, are related to every banknote in the sheet. These parameters are significantly different depending on each denomination.

The inspection algorithm is performed in two different stages:

\section{1: Off-line stage.}

In this stage the system is initialized with the needed conditions for the following on-line stage. In this way, it is necessary to have available information about every printing step. The system performs valid image acquisition of sheets which have the following features:

- Sheets with only watermark.
- Sheets with only intaglio printing.

- Sheets with only offset printing.

- Sheet with both, intaglio and offset printing.

This images will be used in the following on-line stage for the creation of the 'golden images'.

Once this golden images have been created, it is necessary to define specific regions and features of the banknote (printing items). These items are defined in intaglio as well as in offset printings, and they will allow the detection of the position (horizontal and vertical) of each printing. Finding the exact position of every one of these items is performed by means of subpixel procedures in order to achieve a higher accuracy. Several algorithms have been taken into account to find the real position of the step edges that some times have the chosen items. Detecting with subpixel accuracy the zero-crossings [4] of the step edges (the position of certain items) is inadequate due to the high processing time that this algorithm needs, longer than the available time for this step, taking in account that this must be an on-line process. In the same way, the algorithm based in the detection of the position of the items with a momentbased edge operator [6][7], didn't achieve better results than the finally used algorithms, various of them never seen before (pure transition, centre of gravity, increase centre of gravity, etc.).

This algorithms produce different results if they are employed with fixed or variable thresholds, hysteresis cycles, ... In this off-line stage it is also required to realize the optimization of the computer resources of the system, allowing a better performance of the programmed tasks for each denomination, as detailed in the following section (3).

\section{2: On-line stage.}

In the on-line stage the tasks are performed parallely for every banknote among the different acquisition and processing image boards which are present in the system. The tasks can be arranged as:

- Image acquisition.

- Finding items with subpixel accuracy, both intaglio and offset items.

- Generation of the golden images taking in account the position of the intaglio and offset items previously calculated. The system generates two golden images, one to detect the lack defects, and other to detect the excess defects.

- Evaluation of the previous flaws by means of intensity (higher intensity - more severe defect), quantity and proximity.

- Final evaluation of the banknote goodness according to the parameters fixed in off-line stage. 


\section{3: Parallel image processing.}

Before explaining the application it is worth to consider the advantages offered by this kind of system. The main advantage that this system bring is the tradeoff between resolution and real time. One of the main problems originated by the application of computer vision techniques is the huge amount of operations that must be performed on big data sets (images), however presenting sometimes the paradox of having still an insufficient resolution [9].

Presently the choice which is best considerated is to provide structures to the system that allow a parallel processing. It is well known that the SIMD architecture (Single Instruction Multiple Data) has one control unit that gives the instructions for all the processing elements. The given instruction is executed by every processor. Each processor is responsible of a specific section of the complete image, thus each of them can process, on different data, the same operation simultaneously [5].

\section{1: Raising of the problem.}

The large amount of data handled in this application, besides the strong requirements in the production cadence, demands the use of parallel processing image algorithms. The inspection algorithm includes high and low level processings. In order to minimize the processing time, it is needed to use several improvement strategies:

- In some low level processing, as operations point by point between images, the complete image is divided into regions which will be processed by each acquisition and processing image board. All of them are working in parallel.

- Other low level processings, as the reading of some areas of the image, involve the joint use of the CPU and some acquisition and processing image board. In these cases, it is necessary the optimization of the computer resources of the system.

- The high level processings have to be done by the CPU, coexisting with the previous step.

It can be deduced from the previous paragraphs the necessity of making a suitable space and time distribution of the tasks among the existing processors in the system, with the purpose of achieving the optimization of the application. It is important to emphasize that this assignment is going to depend on the inspected denomination. Due to this reason the assignment must be flexible.
In the adopted solution the complete module has the following equipment:

- Processors:

- Inspection unit CPU.

- Processor of board number 1.

- Processor of board number 2.

- Processor of board number 3.

- Processor of board number 4.

- Tasks to be made. The following types of tasks can be distinguished (Table 1):

- Basic processings on the image. They require a small initial use of the CPU (order transference), and then a larger use of the acquisition and processing image board.

- Chaining of basic processings on the image. The basic operations are jointly transferred with the aim of achieving a better development of the utilized resources.

- Reading image zones. It require the joint use of the $\mathrm{CPU}$ and the acquisition and processing image board.

- High or low level calculations. They only use the system CPU.

\begin{tabular}{|c|c|}
\hline OPERATIONS & InIII CPU TIme Board Tme \\
\hline Bastc Procesesing & \\
\hline Baslc Procosoling Chainlng & 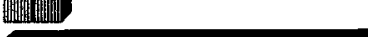 \\
\hline Dema Reading & 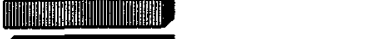 \\
\hline Calculditions & 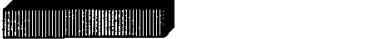 \\
\hline
\end{tabular}

Table 1

\section{2: Adopted solution.}

Initially, it is advisable to explain that the adopted solution solves the problem, minimizing the total processing time of the inspection algorithm for each denomination. The application has the following special features:

- The time provided for the basic image processing is higher than the time that one single board would employ. This requires a division of the complete image in regions, that are handled parallely by each processing board. The number of boards needed to 
adjust the processing time is four.

- The processings in every board are balanced, so there is no need to share images among them.

- The system CPU needs to know the data from the analysis made by each processing board, so a centralized control must be implemented. It wasn't found necessary the use of several CPU's.

- The processing times, both CPU and board times, can be provided previously for each analyzed denomination.

- Inside each board, processings that must not be realized sequentially will exist: e.g. operations between different banknotes.

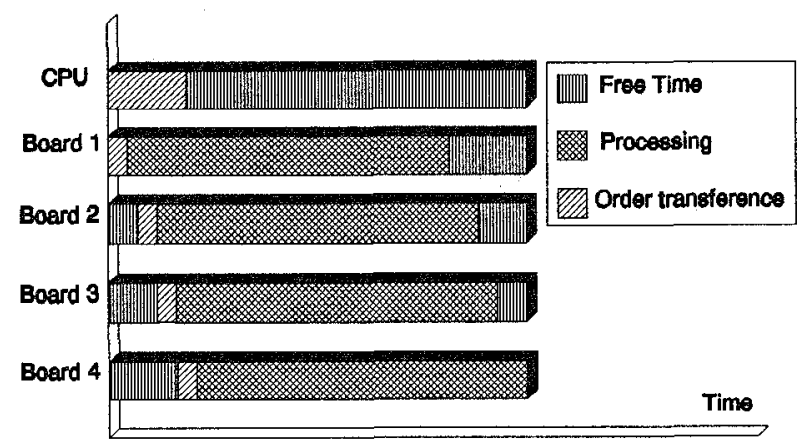

Table 2

The inspection algorithm, from the processing structure point of view, it is carried out in the following steps, all of them off-line:

- The complete image (sheet), is divided into regions with a relative independence. Each region is managed by an acquisition and processing image board. Their distribution is fixed and it depends on the camera set lay-out and its assignment among the boards. Any basic processing will be realized parallely among the four processing boards. This can be seen in the table 2 . Initially the CPU sends the processing order to each board, jointly with its parameters. Then every board begins to work, while the CPU can perform other tasks. The synchronization with the operation end is achieved inquiring the board. Since the processings in each board are the same, it is possible to assume in the future that only one processing unit exits [11].

- For each denomination, the banknote boundaries are fixed as well as the items used in the creation of the golden image. The algorithm produces automatically a task list, as described in table 1 (around 40 tasks per banknote, i.e. 1000 tasks per sheet). The system generates similarly the following data sets:

- For each task it estimates the processing time, in the CPU as well as in the processing boards.

- The total working time for each processor, with the aim of deciding what is the unit with a more critical operation.

- For each task, the jobs that must have been finished previously are listed (e.g. to generate the golden image of a banknote it is necessary to know the positions of the intaglio and offset items of this banknote, but not the positions of the items of the rest of the banknotes in the same sheet).

- Regarding the data previously obtained the tasks to be performed by every processor are assigned in space and time, and so the system resources can be optimized .

The inspection algorithm performs, on-line, the execution of the programmed tasks, in the same order for a specific denomination.

\section{4: Results.}

The system has been tested in the production line with more than 100.000 sheets in the 5.000 pts denomination (legal banknote in Spain). The achieved performance is the following:

- Processing speed of 1 sheet per second.

- Fixation of the alignment, for each side, between the offset an intaglio printings, with an accuracy of $\pm 0.1 \mathrm{~mm}$.

- Fixation of the alignment between front and back intaglio printings with an accuracy of $\pm 0.1 \mathrm{~mm}$.

- Fixation of the intaglio printing location of each banknote with an accuracy of $\pm 0.2 \mathrm{~mm}$. in both axes.

- Fixation of the offset printing location of each note with an accuracy of $\pm 0.2 \mathrm{~mm}$, just for the horizontal axe.

- Fixation, separately, of the presence of the three signatures of each note and the footnote: the system considers each of them present if there exists a $75 \%$ of the item.

- The presence of the security thread in the top and bottom sheet boundaries.

-Spots inkstain. The designed algorithms calculate the number of pixels with spot inkstain on the image and their intensity, rejecting the note according to the off-line defined parameters. The maximum accuracy is the detection of spots inkstain with an intensity of $15 \%$ and a size of $1 \mathrm{~mm} \times 1 \mathrm{~mm}$.

-Lacks of printing. They are calculated similarly, the number of pixels with lacks of printing and their intensities, rejecting the note according to the parameters defined off-line. The maximum accuracy is the same as 
the spots inkstain case.

\section{References:}

[1] Daniel G. Antzoulatos, "Hypermatrix Algebra: Theory", CVGIP: Image Understanding, Vol.57, No.1, January, pp 24-41,1993.

[2] Daniel G. Antzoulatos, "Hypermatrix Algebra. Applications in Parallel Image Processing", CVGIP: Image Understanding, Vol.57, No.1, January, pp 42 63,1993.

[3] Hugo Embrechts, Dirk Roose, Patrick Wambacq, "Component labelling on a MIMD Multiprocessor", CVGIP: Image Understanding, Vol.57, No.2,March, pp 155-165,1993.

[4] Andres Huertas \& Gerard Medioni, "Detection of Intensity Changes with Subpixel Accuracy Using Laplacian-Gaussian Masks", IEEE Transactions on Pattern Analysis and Machine Intelligence Vol. PAMI-8 No.5, Sept. 1986
[5] Zahid Hussain, "Digital Image Processing, practical applications of parallel processing techniques", Ellis Horwood Limited, 1991.

[6] David Lee, G. Wasilkowski, Rajiv Mehrotra, "A New Zero-Crossing-Based Discontinuity Detector", IEEE Transactions on Image Processing Vol. 2 No.2, April,1993.

[7] Edward P. Lyvers, O. Robert Mitchell, Mark L. Akey \& Anthony P. Reeves, "Subpixel measurement using a moment-based edge operator", IEEE Transactions on Pattern Analysis and Machine Intelligence Vol. PAMI-11 No.12, Dec. 1989.

[8] "Image Series", Matrox, 1991.

[9] Anthony P. Reeves, "Parallel Computer Architecturesfor Image Processing", Computer Vision, Graphics, and image processing 25, 68-88 (1984).

[10] J.M ${ }^{\text {a }}$ Sebastián, F.Torres, C. Montes, F. Pisonero, J.L.Bello, E. Barroso, "Revisión Automática de Pliegos por Visión Artificial", $3^{\circ}$ Congreso de la Asociación Española de Robótica (AER), Nov,1993.

[11] F. Torres, J.M ${ }^{a}$.Sebastián, L.M.Jimeméz, "Paralelismo entre Tarjetas de Adquisición de Imágenes", XIV Jornadas de Automática, Sep.1993. 